\title{
Erosion de cavitation d'alliages amortissants à base de manganèse et de cuivre
}

\section{Cavitation Erosion of High Damping Manganese and Copper Based Alloys}

\author{
A. Karimi \\ Institut de Génie Atomique, \\ Ecole Polytechnique Fédérale de Lausanne, \\ 1015 Lausanne (Suisse)
}

\section{Introduction}

Le phénomène de cavitation, par sa nature transitoire, impose aux matériaux un mode de sollicitation du type "contraintes répétitives pulsées" qui peut induire un niveau de contrainte interne élevé dans le réseau cristallin. Ce niveau élevé de contrainte provoque généralement des transformations ou des restitutions structurales qui sont considérées comme bénéfiques pour la résistance à l'érosion de cavitation des alliages [1, 2].

On peut distinguer deux types de transformations en fonction de la valeur de l'énergie de défaut d'empilement $[3,4]$ des matériaux :

- dans les alliages à faible énergie de défaut d'empilement où la déformation se produit par la multiplication des dislocations rectilignes et des défauts planaires, la restitution structurale se produit par micromaclage et transformation de phase;

- dans les alliages à forte énergie de défaut d'empilement la microstructure est constituée par un réseau tridimensionnel de dislocations et la restitution structurale se développe sous forme de recristallisation dynamique.

L'effet bénéfique de ces types de restitutions a été attribué généralement ả la relaxation du cristal et à une meilleure distribution des contraintes internes. Ces observations permettent de s'interroger sur l'effet éventuel d'un autre type de restitution qui se produit dans les alliages dits

\author{
J.L. Heuzé \\ Service Technique des Constructions et Armes Navales, \\ Groupe M.S.N., 8, bd Victor, \\ 75732 Paris Cedex 15
}

amortissants. On sait que dans ces alliages, l'énergie de déformation est en partie dissipée sous forme de chaleur et les mécanismes d'amortissement qui y sont associés sont du même type que le maclage et la transformation martensitique [5].

Cette étude avait pour but essentiel de caractériser la résistance à l'érosion de cavitation de matériaux amortissants, c'est-à-dire capable d'absorber une certaine quantité d'énergie et de n'en restituer qu'une partie via une transformation de phase interne. Mais nous avons également cherché à savoir s'il y avait interaction entre les phénomènes d'amortissement et d'érosion de cavitation de manière à essayer de préciser la nature des influences d'un phénomène sur l'autre.

Ainsi, les questions auxquelles nous souhaitions répondre étaient les suivantes :

- le phénomène d'amortissement a-t-il un rôle actif et bénéfique sur la résistance du matériau à l'érosion de cavitation?

- la cavitation, par sa nature transitoire sous la forme de pics de pression localisés mais d'amplitude élevée, ne conduit-elle pas à diminuer, voir à supprimer le caractère amortissant du matériau?

Cette publication présente les premiers résultats obtenus à l'aide de différentes techniques telles que la microscopie électronique (MET, MEB), la diffraction des rayons $\mathrm{X}$ et la micro-indentation.

\section{Cavitation erosion of high damping maganese and copper based alloys}

High damping capacity of $\mathrm{Mn}$ and $\mathrm{Cu}$ based alloys have been subjected to cavitation erosion in a vortex generator. These alloys show a moderate cavitation erosion resistance, close to Cu Al9 alloy. The experimental results revealed a better resistance of $\mathrm{Cu}-\mathrm{Mn}$ alloys compared with $\mathrm{Mn}-\mathrm{Cu}$ alloys. The dendritic phase rich in iron and heterogeneous is unfavourable for cavitation erosion resistance. Micro-twins considered at the origin of damping mechanisms seem to be favourable to the erosion resistance. Strain hardening capacity, ductility and fatigue limit of the matrix phase are the most important parameters to control cavitation erosion resistance of high damping alloys. 
Tableau 1:

Composition chimique

\begin{tabular}{|l|c|c|c|c|c|c|c|}
\hline & \multicolumn{6}{|c|}{ ELEMENTS 5 EN POIDS } \\
\hline Matćriaux & Mn & Cu & Al & Fe & Ni & Si & C \\
\hline $\begin{array}{l}\text { Sonoston } \\
\text { type SH }\end{array}$ & 51.2 & 38.4 & 4.2 & 4.9 & 1.6 & 0.05 & 0.07 \\
\hline $\begin{array}{l}\text { Sonoston } \\
\text { type S }\end{array}$ & 55.6 & 34.0 & 4.4 & 4.5 & 1.5 & 0.14 & 0.012 \\
\hline Incramute IN & 41.0 & 56.2 & 1.4 & 1.2 & 0.1 & 0.09 & 0.018 \\
\hline
\end{tabular}

Tableau 2 :

Caractéristiques mécaniques

\begin{tabular}{|l|l|l|l|l|}
\hline Propriétés & & $\begin{array}{l}\text { Sonoston } \\
\text { type SH }\end{array}$ & $\begin{array}{l}\text { Sonoston } \\
\text { type S }\end{array}$ & $\begin{array}{l}\text { Incramute } \\
\text { IN }\end{array}$ \\
\hline Rp 0.2 & Mpa & 289 & 305 & 220 \\
\hline Rm & Mpa & 458 & 439 & 540 \\
\hline A & $\%$ & 18 & 18 & 31 \\
\hline E & Mpa & 67000 & 61000 & 89000 \\
\hline KCU & J.cm ${ }^{-2}$ & 35 & ND** & ND \\
\hline $\begin{array}{l}\text { Dureté } \\
\text { matrice }\end{array}$ & $\begin{array}{l}\text { HV } \\
\text { min }\end{array}$ & 167 & 156 & 130 \\
\hline $\begin{array}{l}\text { oD } \\
10^{7} \\
\text { cycles }\end{array}$ & Mpa (Air) & 35 & ND & ND \\
\hline mmmmmmmm & & $<20$ & 30 & 65 \\
\hline $\begin{array}{l}* \\
\text { OD (A3) }\end{array}$ & & 0.07 & 0.10 & 0.30 \\
\hline Rp0,2 & - & & & \\
\hline
\end{tabular}

- Eau de mer synthétique

** ND: Non déterminé

Tableau 3:

Mesure de l'amortissement

\begin{tabular}{|l|c|c|c|c|}
\hline Matériaux & $\begin{array}{c}\text { charge } \\
\mathrm{Mpa}\end{array}$ & $\begin{array}{c}\mathrm{W} \\
\mathrm{KJ}\end{array}$ & $\begin{array}{c}\mathrm{Q}^{1} \\
\%\end{array}$ & $\begin{array}{c}\Delta \mathrm{W} \\
\mathrm{KJ}\end{array}$ \\
\hline \multirow{3}{*}{ Sonoston } & \pm 30 & 9.7 & 8.2 & 5 \\
$\mathrm{SH}$ & \pm 50 & 27 & 8.5 & 15 \\
& \pm 100 & 96 & 6.0 & 36 \\
\hline \multirow{2}{*}{ Sonos- } & \pm 30 & 6.2 & 4.3 & 1.7 \\
témoin & \pm 100 & 71 & 4.3 & 4.1 \\
& & & & 20.0 \\
\hline & & & & \\
Incramute & \pm 35 & 9.6 & 4.5 & 2.7 \\
\hline
\end{tabular}

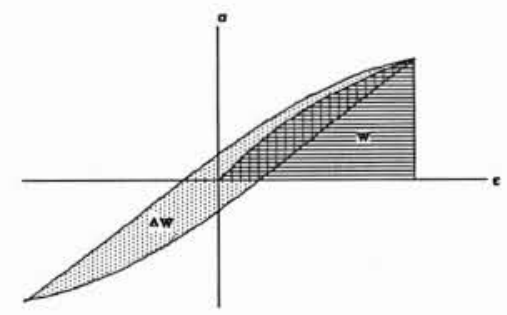

2. Boucle d'hystérésis du diagramme contrainte - déformation et définition de la capacité spécifique d'amortissement.
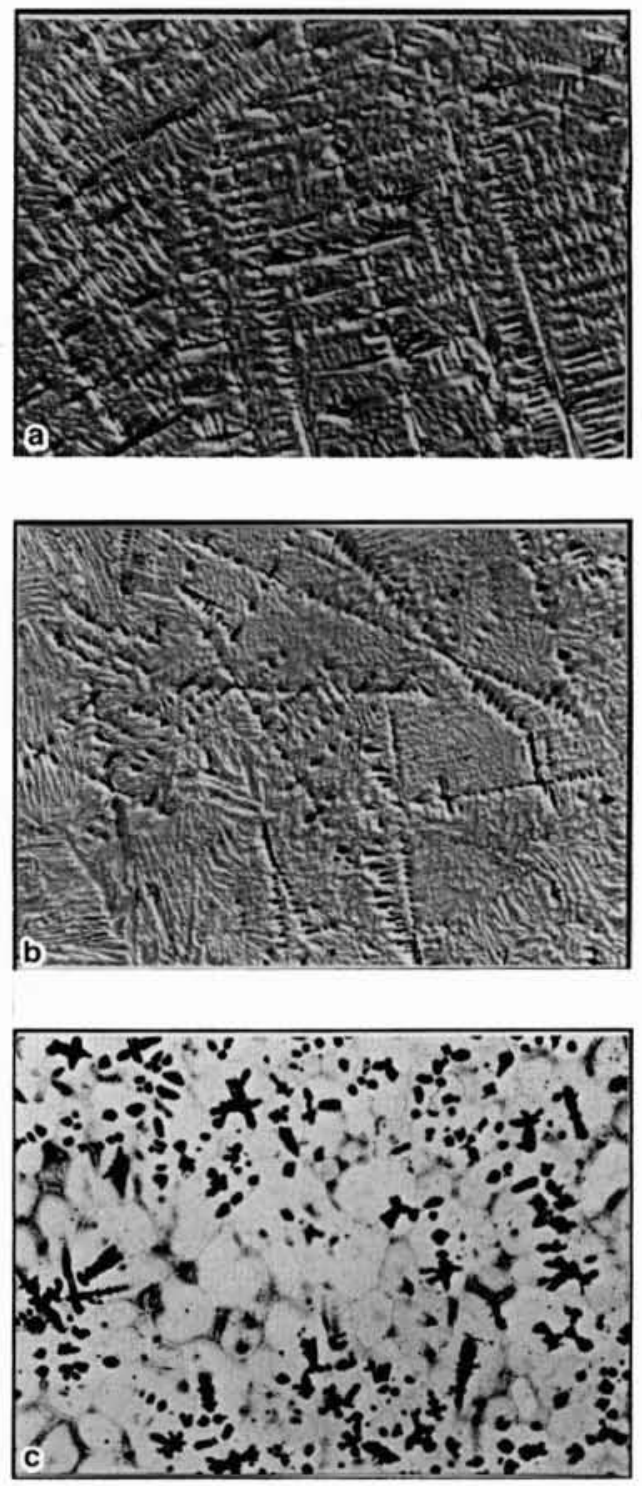

1. Structure des alliages amortissants des différentes nuances. a) Sonoston $S H$, b) Sonostémoin $S$, c) Incramute $I N$.
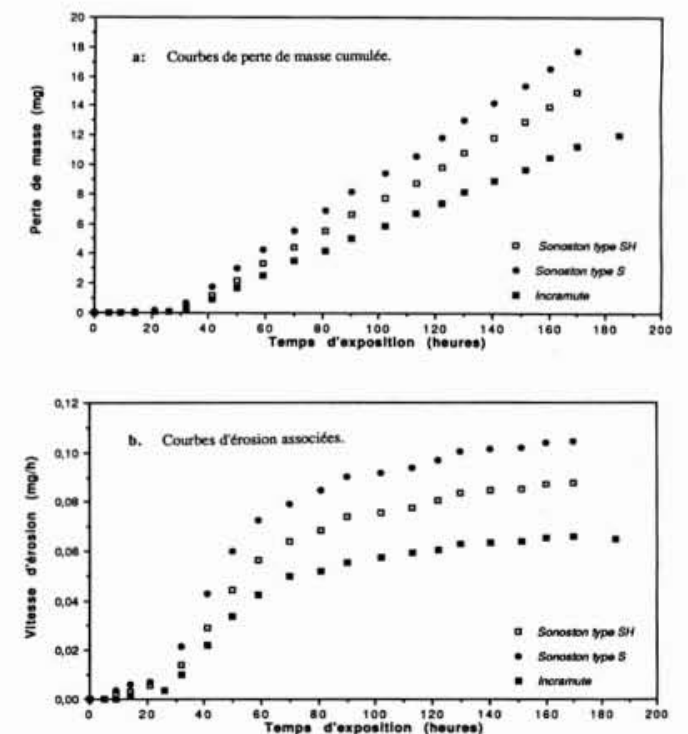

3. Courbes d'érosion obtenues dans la veine tourbillon pour différents matériaux.

a) Courbes de perte de masse, b) Courbes d'érosion associées. 


\section{Matériaux étudiés}

L'étude a porté sur le comportement de nuances voisines d'alliages à base de manganèse et de cuivre. Le tableau 1 présente la composition chimique des 3 matériaux étudiés.

Pour deux d'entre eux, les matériaux repérés $\mathrm{S}$ et $\mathrm{SH}$, on notera qu'il s'agit d'alliages base manganèse plus connus sous l'appellation commerciale de SONOSTON. Cette nuance a été mise au point par la société Stone Marine Manganèse [6].

Le dernier alliage est une base cuivre très chargée en manganèse $(>40 \%)$. Il est désigné sous l'appellation INCRAMUTE et a été mis au point par l'International Copper Research Association (INCRA).

La composition chimique de ces 3 alliages est présentée dans le tableau 1 , tandis que les caractéristiques mécaniques associées font l'objet du tableau 2.

Les cibles de cavitation sont des pièces cylindriques de $12 \mathrm{~mm}$ de diamètre et $11 \mathrm{~mm}$ de hauteur. Elles ont été usinées dans du rond de $80 \mathrm{~mm}$ provenant de la société Coyard pour le Sonoston type S et pour l'Incramute, alors que pour le Sonoston type $\mathrm{SH}$, elles sont issues d'une pièce moulée par les fonderies phocéennes. La microstructure des 3 alliages est présentée figure 1 .

Les deux nuances de Sonoston $\mathrm{S}$ et $\mathrm{SH}$ présentent, à l'échelle quasi macroscopique, une structure particulière constituée de dendrites sombres noyées dans une matrice à l'aspect " écaillé » ( $(f i g . l a$ et $l b)$. L'Incramute lui présente une structure plus traditionnelle constituée par des grains de phase $\alpha$ à l'intérieur desquels on observe une phase dendritique de couleur sombre riche en manganèse (fig. Ic). On constate, cà et là, la présence de quelques micro-retassures dont la taille toutefois ne dépasse pas $50 \mu \mathrm{m}$.

\section{Capacité d'amortissement}

L'amortissement est une propriété particulièrement sensible, en étroite relation avec les autres caractéristiques du matériau. La propriété que possède un solide, en vibration, de dissiper de l'énergie (généralement sous forme de chaleur) est désignée sous le nom de capacité d'amortissement ou de frottement interne ou de frottement intérieur [7].

La capacité spécifique d'amortissement est le rapport entre l'énergie dissipée au cours d'un cycle $(\Delta \mathrm{W})$ et l'énergie élastique maximale emmagasinée au cours du cycle (W) (fig. 2).

Cette définition est générale. Par contre, les valeurs mesurées dépendent du mode et de la forme des sollicitations et des réponses, c'est-à-dire du mécanisme d'amortissement.

$$
\Psi=\frac{\Delta W}{W}
$$

Il existe plusieurs méthodes de mesure de la capacité d'amortissant d'un matériau [7], les 2 principales étant :

- la mesure du frottement intérieur par pendule,
- la mesure des hystérésis sous sollicitations cycliques de fatigue.

C'est cette dernière méthode qui a été utilisée pour mesurer l'amortissement sur les 3 matériaux. Les résultats obtenus sont regroupés dans le tableau 3. Dans ce tableau $\mathrm{Q}^{-1}$ est défini par la relation $\mathrm{Q}^{-1}=\Psi / 2 \pi$

\section{Résultats}

\section{Courbes d'érosion}

Les essais d'érosion ont été menés pour des durées allant de 1 minute à 180 heures. Les essais de courte durée étaient plus particulièrement destinés à l'étude métallurgique.

Deux types de courbes d'érosion sont présentés :

- courbes d'érosion en perte de masse cumulée: $\Delta \mathrm{m}=\mathrm{f}(\mathrm{t})$ (fig. $3 a)$;

- courbes d'érosion en vitesse d'érosion : $\mathrm{e}=\Delta \mathrm{m} / \mathrm{t}=\mathrm{g}(\mathrm{t})($ fig. $3 b)$.

Les courbes d'érosion obtenues (fig. 3), présentent les 4 périodes distinctes et habituelles des courbes d'érosion:

- période d'incubation pendant laquelle l'échantillon est essentiellement écrouit, la perte de masse est négligeable en raison du comportement ductile du matériau. La durée de cette période est d'environ 20 heures pour le Sonoston et 24 heures pour l'Incramute

— période d'accélération où l'écrouissage progressif s'approche de la saturation $\varepsilon_{\mathrm{R}}$ et le taux d'érosion augmente rapidement pour atteindre un maximum. Le déchaussement préférentiel des précipités dans les dendrites favorise encore l'érosion au cours de cette période;

- la période stationnaire est un plateau où le taux d'érosion reste pratiquement constant, $\delta \mathrm{e} / \delta \mathrm{t}=0,105 \mathrm{mg} / \mathrm{h}$ pour le Sonoston $\mathrm{S}$ et $\delta \mathrm{e} / \delta \mathrm{t}=0,085 \mathrm{mg} / \mathrm{h}$ pour le Sonoston $\mathrm{SH}$ et $\delta \mathrm{e} / \delta \mathrm{t}=0,065 \mathrm{mg} / \mathrm{h}$ pour l'Incramute

- finalement, la période d'atténuation, qui correspond à un ralentissement du taux d'érosion, soit en raison de l'accommodation entre l'état de surface de l'échantillon et l'écoulement, soit en raison de la baisse de la dynamique de vortex. En effet, au fur et à mesure de l'érosion, la surface exposée s'éloigne du centre de formation de vortex, ce qui conduit à une diminution de l'intensité des impacts.

Les résultats expérimentaux sont regroupés dans le tableau 4 (page suivante).

Nous constatons, d'une part que l'Incramute, alliage $\mathrm{Cu}-\mathrm{Mn}$, résiste mieux à l'érosion de cavitation que les 2 nuances de Sonoston et d'autre part, que parmi les 2 nuances de Sonoston étudiées le Sonoston $\mathrm{SH}$ résiste un peu mieux que le Sonoston S.

\section{Comparaison avec d'autres matériaux}

Afin de montrer de manière plus explicite le comportement à l'érosion de cavitation des alliages amortissants étudiés, nous les avons reportés dans l'histogramme de la figure 4. 
Tableau 4:

Résultats expérimentaux d'érosion

\begin{tabular}{|l|c|c|c|c|}
\hline Alliage & ti (h) & $e_{\max }$ & $\begin{array}{c}\text { Densité } \\
\left(\mathrm{g} / \mathrm{cm}^{3}\right)\end{array}$ & $\begin{array}{c}\mathrm{e}_{m_{\text {mg }}} \\
\left(\mathrm{mm}^{\mathrm{h}} \mathrm{h}\right)\end{array}$ \\
\hline $\begin{array}{l}\text { Sonoston } \\
\text { type S }\end{array}$ & 20 & 0.105 & 7.78 & 0.0135 \\
\hline $\begin{array}{l}\text { Sonoston } \\
\text { type S }\end{array}$ & 20 & 0.085 & 7.88 & 0.0108 \\
\hline Incramute & 24 & 0.065 & 8.22 & 0.0079 \\
\hline
\end{tabular}

Tableau 5:

Paramètres de microdureté de la matrice

\begin{tabular}{|l|c|c|c|c|c|}
\hline Alliage & $\begin{array}{l}\text { HV } \\
\mathrm{min}\end{array}$ & $\begin{array}{l}\text { HV } \\
\max \end{array}$ & $\begin{array}{l}\text { HV } \\
(\max - \\
\min )\end{array}$ & $\begin{array}{l}\text { HV maxi } \\
\text { HVini }\end{array}$ & $\begin{array}{l}\text { Profondeur } \\
\mu \mathrm{m}\end{array}$ \\
\hline Sonoston & 156 & 330 & 174 & 2.12 & 600 \\
\hline Incramute & 130 & 280 & 150 & 2.15 & 400 \\
\hline
\end{tabular}

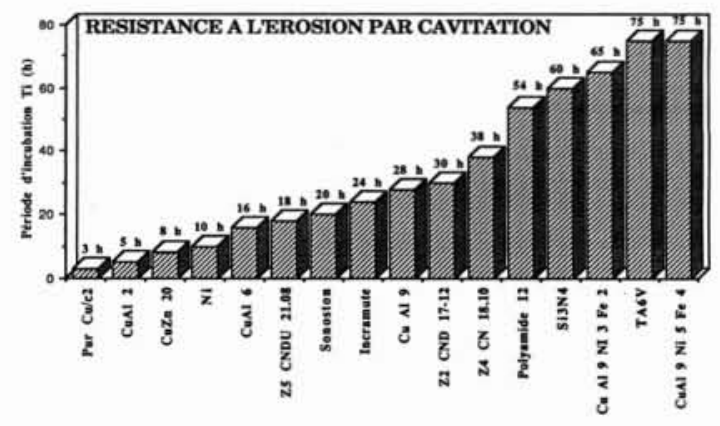

4. Histogramme de comparaison de la résistance à la cavitation de plusieurs alliages industriels.

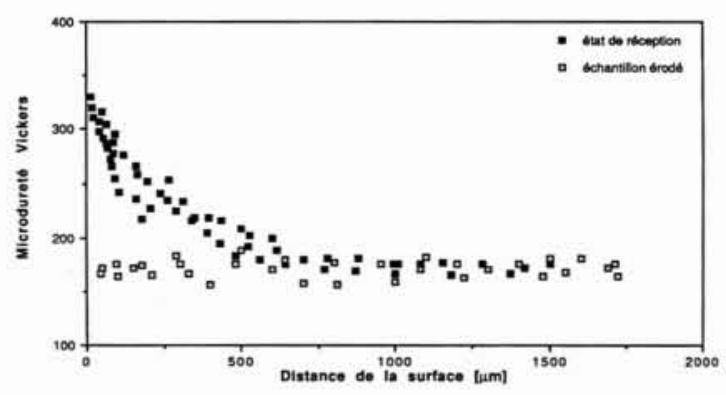

5. Microdureté sur coupe transversale des échantillons érodés.
La classification effectuée à partir des périodes d'incubation des matériaux montre que ces alliages ont une résistance comparable à des alliages $\mathrm{CuAl}$ faiblement alliés du type CuAl6 et CuAl9.

Si la comparaison avait été effectuée à partir du taux maximal d'érosion obtenu en mode stationnaire, la physionomie du diagramme de la figure 4 serait restée pratiquement inchangée.

\section{Evolution de la microdureté}

Deux types de mesure de microdureté ont été effectués : - avant cavitation, sur la surface de l'échantillon, afin d'évaluer la dureté respective des différentes phases présentes ;

- après cavitation, suivant une coupe longitudinale passant par le centre de la zone érodée de l'échantillon. Nous avons alors mesuré l'évolution de la dureté en fonction de la distance par rapport à la surface érodée afin d'évaluer la profondeur de la zone écrouie. Les valeurs relevées pour la matrice figurent dans le tableau 5 :

- Sonoston

- dendrites :

- précipités :

128 à $146 \mathrm{HV}$

- Incramute

- dendrites :

185 à $195 \mathrm{HV}$

La plus faible dureté des dendrites peut s'expliquer par le fait que les précipités ne forment pas un milieu compact et homogène. On notera que les dendrites dans l'Incramute sont moins dures que celles du Sonoston.

Les plages de dureté relativement larges de l'Incramute $(\mathrm{Hv}=710-162)$ et du Sonoston $(\mathrm{Hv}=1280-0195)$ mettent en évidence l'hétérogénéité de la structure de ces matériaux.

Les résultats des profils de microdureté du Sonoston, après cavitation, suivant une coupe longitudinale sont présentés figure 5.

Il faut noter qu'ils correspondent uniquement à la dureté de la matrice. D'après ces résultats, la profondeur d'écrouissage peut être estimée à environ $650-700 \mu \mathrm{m}$, et le rapport de la dureté maximale (à la surface érodée) sur la dureté d'origine est $\mathrm{HVmax} / \mathrm{HVo} 2,1$.

\section{Examen de l'endommagement et mécanisme d'érosion}

La figure 6 présente quelques aspects essentiels des faciès d'érosion des nuances de Sonoston à comparer avec ceux de l'Incramute observés figure 7.

Pour analyser la dégradation, par érosion de cavitation, d'un matériau hétérogène comme le Sonoston, il convient de distinguer le comportement des 2 constituants principaux : la matrice d'une part, et les dendrites d'autre part. Ainsi, l'observation des impacts isolés sur le Sonoston (fig. 6a) met en évidence le caractère ductile de la déformation de la matrice. Cette déformation procède par activation de nombreux systèmes de glissement, au moins 3 (fig. 6 b et c), qui conduisent à la formation de bandes de 

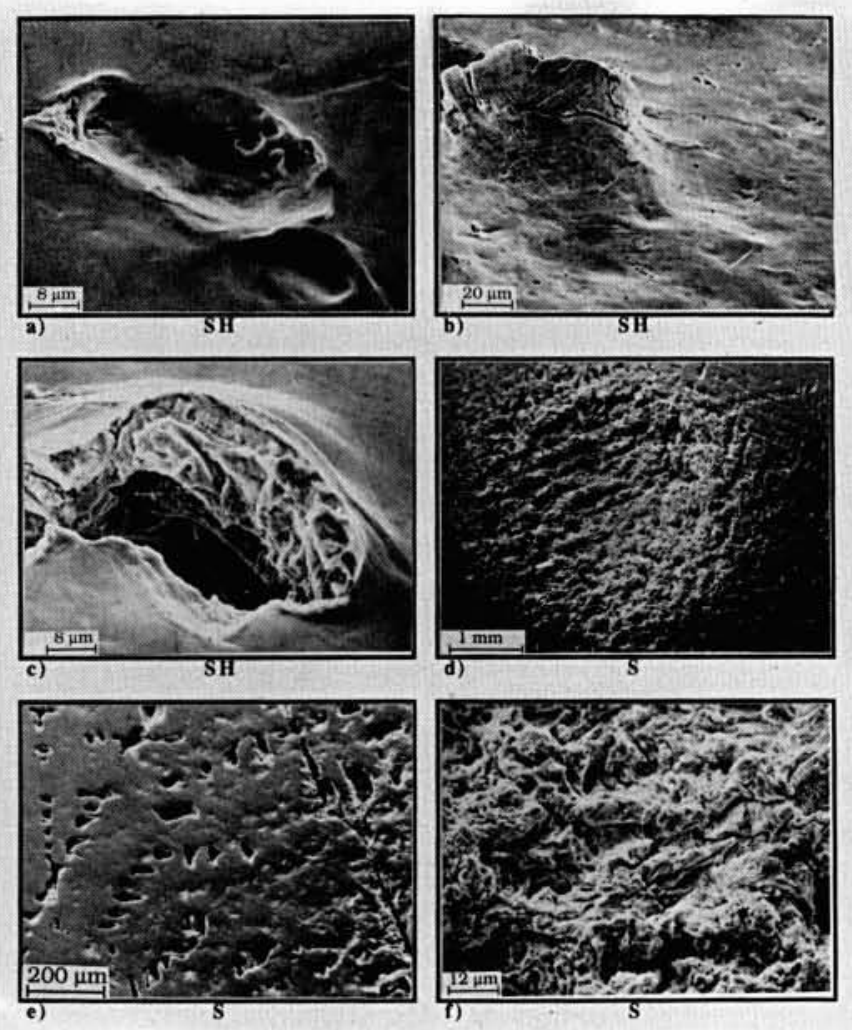

6. Examen au M.E.B. des échantillons de Sonoston. a) impact isolé, b) c) déformation de la surface, d) surface érodée Sonoston, e) érosion préférentielle de dendrités, f) érosion avancée.
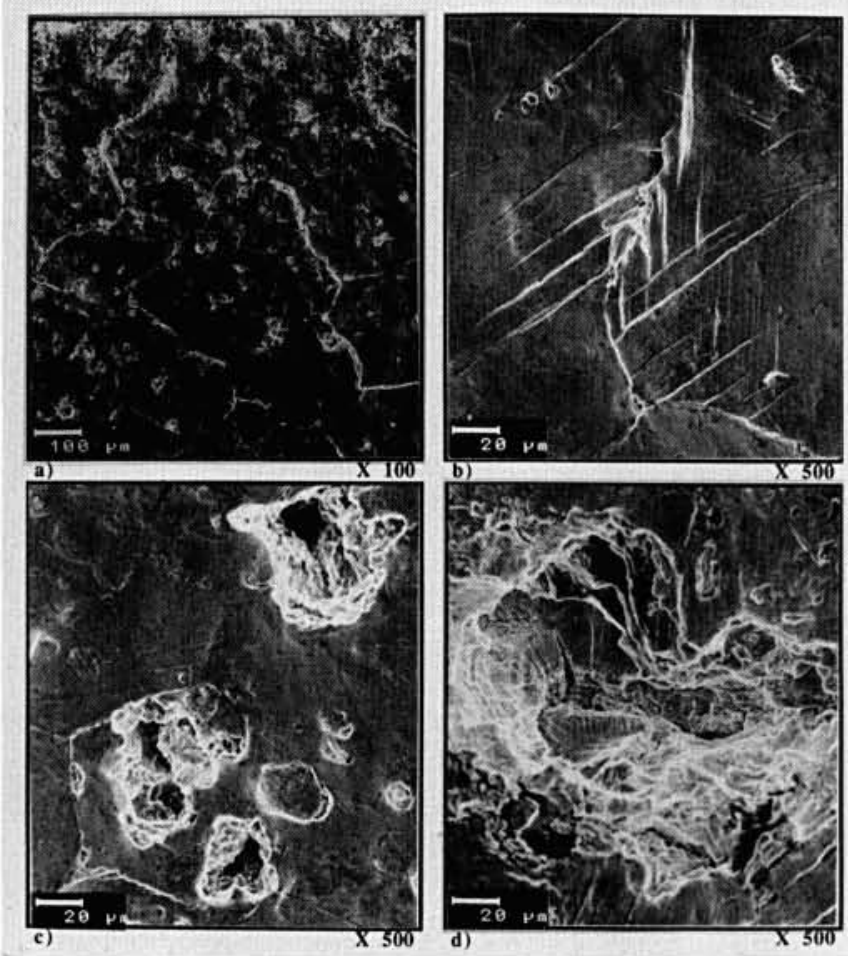

7. Examen au M.E.B. des échantillons d'Incramute.

a) érosion préliminaire, b) déformation de la surface. glissement persistantes; ces dernières constituant, à termes, des sites privilégiés de fissuration (fig. $6 b$ ).

L'examen au M.E.B. d'un échantillon après plus de 100 heures de cavitation (fig. 6 d) permet de constater que l'érosion débute à l'interface matrice/dendrites et se poursuit par l'érosion de la phase dendritique, plus molle mais plus fragile car beaucoup plus hétérogène (fig. $6 c$ et $6 e$ ). Cette érosion des dendrites favorise alors l'implosion du vortex dans les cavités et cratères ainsi créés dans la matrice, conduisant à une déformation considérable de celle-ci. De plus, la forme allongée des dendrites induit, lors des implosions, des concentrations de contraintes importantes au fond de ces macro-fissures, ce qui accélère la fissuration de la matrice.

Au stade de dégradation avancée, nous constatons que l'érosion procède par fissuration progressive et fragmentation en petits morceaux de 10 à $20 \mu \mathrm{m}$ de longueur (fig. $6 f$ ).

Sur l'Incramute, le processus d'endommagement est légèrement différent et pourrait expliquer la meilleure résistance de ce dernier par rapport au Sonoston. L'endommagement semble, d'une part, s'amorcer aux joints de grains qui constituent une des zones les plus fragiles de l'alliage (fig. $7 a$ ) et d'autre part, dans les dendrites (fig. $7 a$ et c) mais leur proportion est nettement plus faible que dans le Sonoston.

La déformation de la matrice procède par glissement avec formation de lignes et de bandes de glissement persistantes (fig. $7 b$ ). Ce sont ces lignes de glissement persistantes qui en s'accentuant sous l'effet des impacts conduisent à la fissuration selon un mécanisme analogue à celui de la fissuration par compression cyclique ondulée (fig. 7d).

Des examens effectués au microscope optique sur coupe transversale d'un échantillon d'Incramute ayant subi $180 \mathrm{~h}$ de cavitation, permettent de mettre en évidence les différents modes de propagation des fissures.

Ainsi, on constate que la fissuration tend à s'amorcer sur le mode intergranulaire puis à se propager très rapidement en mode transgranulaire tout comme les fissures de fatigue obtenues par traction-compression. La sollicitation n'étant pas uniaxiale, homogène et uniforme, on notera d'une part, que les fissures se propagent selon différentes orientations et d'autre part, que ces fissures ont une forte tendance à se ramifier. Ces deux facteurs sont très favorables à la relaxation des contraintes. 

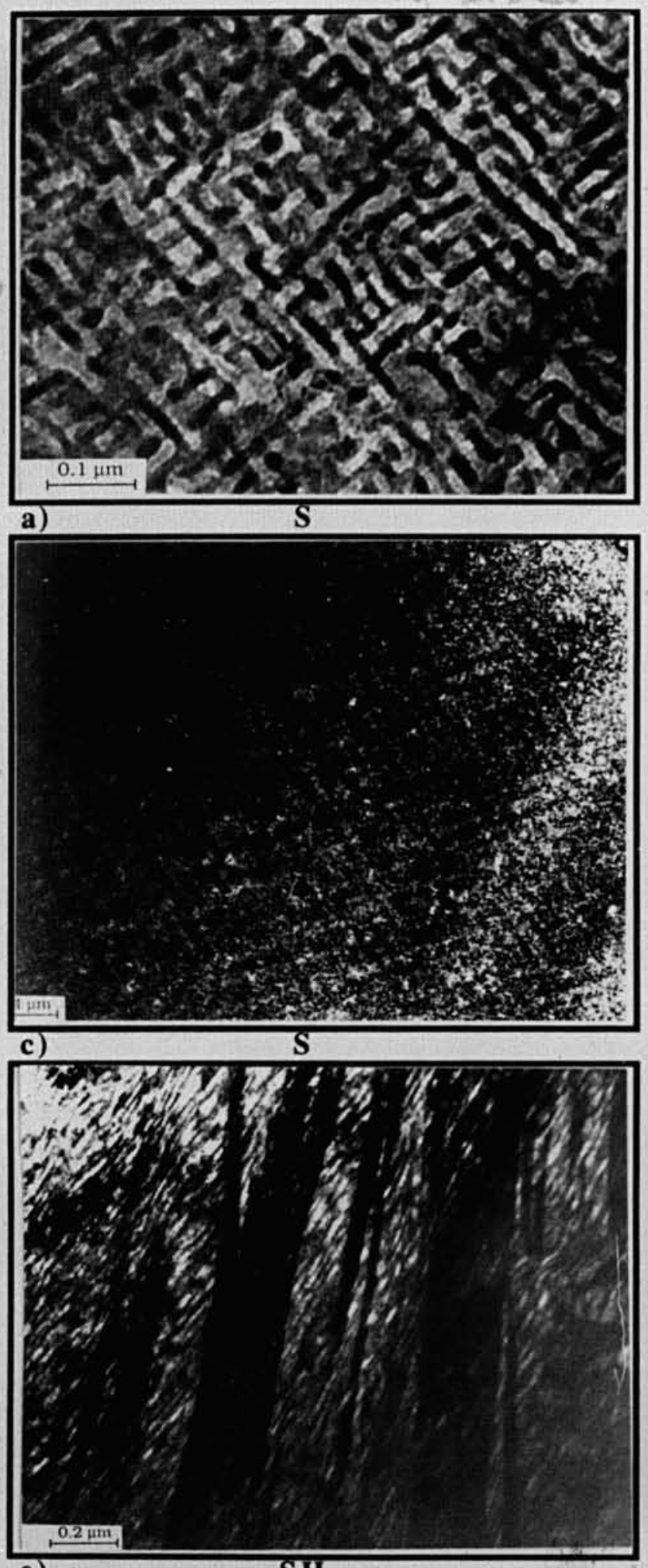

e)

SH
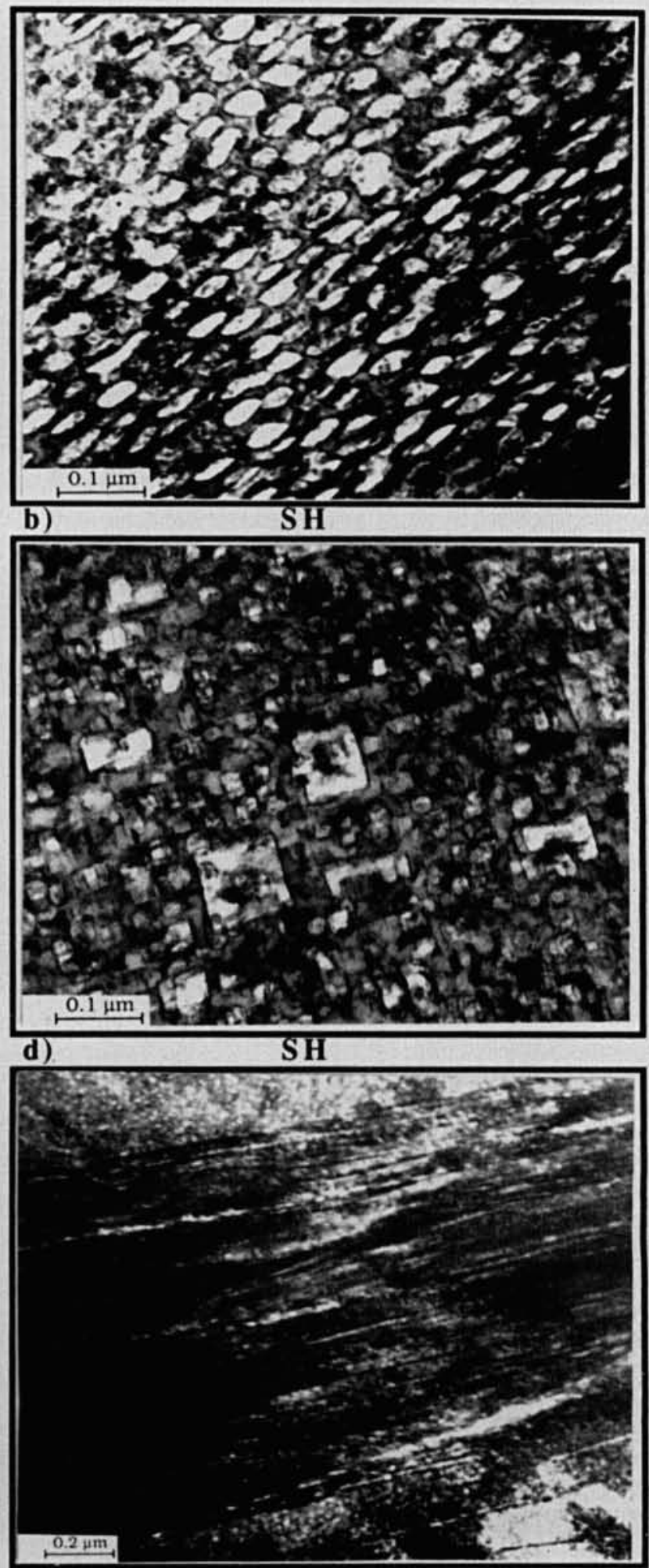

f)

SH

8. Micrographie au microscope électronique à transmission de Sonoston érodé. a - e) Sonoston $S$, b - f) Sonoston $S H$. 


\section{Discussion des résultats}

D'après l'histogramme présenté fig. 4 , nous constatons que les alliages $\mathrm{Mn}-\mathrm{Cu}$ ont une résistance médiocre à l'érosion de cavitation, par rapport aux alliages traditionnellement utilisés pour la fabrication des hélices marines comme les cupro-aluminiums complexes du type $\mathrm{Cu}$ A19 Ni5 Fe4.

Parmi les 3 alliages étudiés, nous notons une différence significative de résistance à l'érosion :

- l'alliage $\mathrm{Cu}-\mathrm{Mn}$ (Incramute) est entre 20 et $40 \%$ plus résistant que les alliages $\mathrm{Mn}-\mathrm{Cu}$ (Sonoston);

- la nuance de Sonoston SH présente une résistance de 15 à $20 \%$ supérieure à la nuance de Sonoston $S$.

Ces différences de comportement sont à relier à :

- la microstructure et en particulier à la fraction volumique de dendrites nettement plus importante dans les alliages $\mathrm{Mn}-\mathrm{Cu}$ que dans les alliages $\mathrm{Cu}-\mathrm{Mn}$ comme l'Incramute ;

- la nature de la matrice. Dans l'Incramute, la matrice est constituée de phase $\alpha$ austénitique de structure C.f.c. cristalline qui accommode la déformation par glissement de la même manière que pour les cupro-aluminiums. De plus, son caractère ductile $(A=31 \%)$ favorise l'accommodation des contraintes donc la résistance à l'érosion.

En revanche, dans les alliages dont le constituant essentiel est le manganèse, on ne retrouve pas ce caractère cristallographique (fig. $1 \mathrm{a}$ et $\mathrm{lb}$ ) et la ductilité plus faible de l'alliage $(18 \%)$ ne favorise pas l'accommodation des contraintes générées par les impacts de cavitation.

Notons enfin la grande différence entre la limite d'endurance du Sonoston SH et celle de l'Incramute. Or le phénomène de cavitation, par sa nature transitoire, impose au matériau un mode de sollicitation de type "contraintes pulsées localisées et répétitives » que l'on peut considérer comme un phénomène de fatigue localisé.

Si l'on comprend aisément pourquoi l'Incramute résiste mieux que le Sonoston à l'érosion de cavitation, il n'en est pas de même pour expliquer les différences observées entre les 2 nuances de Sonoston. En effet, le Sonoston type SH qui résiste mieux, présente une fraction volumique de dendrites plus importante que la nuance $S$, ce qui est défavorable pour la tenue à l'érosion mais confère un pouvoir amortissant plus important. C'est donc vis-à-vis de la résistance de la matrice qu'il faut chercher l'explication et en particulier du côté de la microstructure à l'échelle de la microscopie électronique en transmission (fig. 8). Dans le sonoston type $S$, on observe une structure "tweed" (fig. $8 a$ ) tandis que dans le sonoston type $S H$. la structure est microcristalline lenticulaire ( fig. $8 \mathrm{~b} \mathrm{et} \mathrm{d)} \mathrm{et}$ la densité de micro-macles mécaniques y est plus importante (fig. 8 e et $f$ ) que dans le sonoston type $S$ (fig. $8 \mathrm{c}$ ). Or, nous savons que les micro-macles favorisent la résistance à l'érosion de cavitation [9][10][11].
Mécanisme d'amortissement

Le phénomène d'amortissement dans les métaux provient de mouvements de défauts cristallins ponctuels (atomes, lacunes), linéaires (dislocations), ou plans (joints de grains, macles, parois de domaines magnétiques...). Il n'y a amortissement que si et seulement si ces défauts sont mobiles. De même, tout ce qui modifie le nombre, la nature, ou la répartition des défauts cristallins et des parois de domaines tels que l'écrouissage ou les traitements thermiques, modifie le pouvoir amortissant de l'alliage [12].

Le mécanisme microscopique de l'amortissement a généralement été attribué aux phénomènes suivants :

- la distorsion du réseau qui peut accommoder la déformation par incrément des segments inter-atomiques. En général, l'amortissement ainsi obtenu est faible et souvent négligeable ;

- le mouvement élastique des dislocations qui nécessite une quantité importante d'énergie. Lorsque la contrainte appliquée est inférieure à la limite du décrochement, le mouvement réversible des dislocations peut dissiper l'énergie emmagasinée et amortir la contrainte ;

- la transformation martensitique (pseudoélastique) qui est induite par le mouvement coopératif des atomes sous une contrainte appliquée. L'élimination de la contrainte extérieure permet une réversion atomique et un amortissement relativement important.

- les phénomènes liés aux joints et aux diverses parois, dont le mouvement est considéré comme le mécanisme d'amortissement le plus important, sont :

- les parois des plaquettes de martensite,

- les parois des domaines ferromagnétiquesantiferromagnétiques,

- les parois d'interfaces entre la matrice austénitique et les plaquettes de martensite [13].

Dans le cas des alliages à transformation martensitique comme les alliages $\mathrm{Cu} \mathrm{Al} \mathrm{Ni,} \mathrm{c'est} \mathrm{la} \mathrm{réorganisation} \mathrm{des}$ plaquettes de martensite qui génère l'amortissement [14].

Dans les alliages composés des éléments de transition présentant un fort gradient du moment magnétique tels que $\mathrm{Mn}, \mathrm{Cr}$ et $\mathrm{Fe}$, c'est le mouvement des parois des domaines ferromagnétique-antiferromagnétique qui semble générer l'effet d'amortissement [15].

L'alignement des moments magnétiques dans les domaines modifie la distance inter-atomique du réseau dans la direction de la magnétisation et crée l'expansion locale des grains.

Ce phénomène se manifeste dans les alliages $\mathrm{Mn}-\mathrm{Cu}$, par la transition $\mathrm{Cfc} \rightarrow$ Qfc liée à l'orientation de l'axe $C$ dans des zones riches en $\mathrm{Mn}$ suivant les domaines. Ceci apparaît sur les micrographies TEM par un contraste dynamique de "tremblotement» (fig. $8 b, d$ et e). En raison de la géométrie spécifique des alliages $\mathrm{Mn}-\mathrm{Cu}$, cette transition est équivalente à la formation d'une macle mécanique, ce qui nous permet de suggérer que l'apparition des macles mécaniques dans des alliages $\mathrm{Mn}-\mathrm{Cu}$ (fig. 8 e et f) puissent être associées à leur capacité d'amortissement. 


\section{Influence de la capacité d'amortissement sur la résistance à l'érosion de cavitation}

De nombreuses études [11][16][17] effectuées sur des alliages cuivreux et en particulier les $\mathrm{Cu} \mathrm{Al}$ ont mis en évidence que la résistance à l'érosion de cavitation est fonction du ou des modes de déformation de l'alliage. Ainsi, les matériaux les plus résistants sont ceux, à basse énergie de défauts d'empilement, qui se déforment par maclage. Or, il apparaît que c'est le phénomène de micromaclage qui procure aux matériaux étudiés la plus grande part de leur capacité d'amortissement. On peut donc supposer que les transformations structurales qui concourent à créer et développer la capacité d'amortissement d'un matériau métallique ne peuvent qu'avoir un effet positif sur la résistance à l'érosion de cavitation du matériau. Toutefois, il convient de tempérer cet aspect positif, par le caractère hétérogène de ces matériaux pour lesquels la phase dendritique plus molle a généralement une très mauvaise résistance à l'érosion de cavitation comme l'ont démontré les examens au MEB.

\section{Influence de la cavitation sur le phénomène d'amortissement}

D'après les études de modélisation des matériaux amortissants effectuées par J.C. HAMANN [7], il apparaît que, sous sollicitation cyclique, le degré d'amortissement dépend de l'amplitude de la sollicitation cyclique. Dans un premier temps, la cavitation pourrait fournir l'énergie nécessaire à la transformation de phase comme cela a déjà été observé sur les aciers austénitiques type 304 [11] et générer l'apparition de micromacles augmentant ainsi la capacité d'amortissement du matériau. Néanmoins, la saturation de l'écrouissage et la déformation plastique intense auront à terme pour effet de détruire l'effet amor- tissant comme précisé par C. VERNOT [18]. L'influence de la cavitation sur l'amortissement serait donc directement fonction de l'intensité de celle-ci et du niveau de contrainte qu'elle génère dans le matériau. Si la contrainte appliquée est supérieure à la limite d'endurance du matériau, l'écrouissage progressif de la matrice conduit à la diminution de la capacité d'amortissement par modification des conditions de frottement des interfaces en présence, sans oublier également l'apparition, à terme, de microfissures de fatigue.

\section{Conclusion}

Les alliages $\mathrm{Cu}-\mathrm{Mn}$ comme l'Incramute résistent mieux à l'érosion de cavitation que les alliages $\mathrm{Mn}-\mathrm{Cu}$ tel que le Sonoston. Cette différence est probablement à attribuer à la meilleure résistance de la matrice des alliages $\mathrm{Cu}-\mathrm{Mn}$ par rapport à celle des alliages $\mathrm{Mn}-\mathrm{Cu}$.

Du point de vue métallurgique, la nature hétérogène des alliages $\mathrm{Mn}-\mathrm{Cu}$ et $\mathrm{Cu}-\mathrm{Mn}$ amortissants étudiés conduit globalement à une tenue médiocre à l'érosion de cavitation. Les différences importantes de dureté des phases en présence conduisent à des interfaces peu résistantes aux impacts de cavitation. Nous avons également remarqué que la matrice résiste beaucoup mieux que la phase qualifiée d'amortissante qui est la plupart du temps très hétérogène.

Toutefois le phénomène d'amortissement en lui-même, par les transformations de phases qui le génèrent, ne peut avoir qu'un effet bénéfique sur la résistance à l'érosion de cavitation, mais ce dernier est nettement insuffisant pour contrebalancer l'effet négatif dû à l'hétérogénéité de la structure.

\section{Références bibliographiques}

[1] S. Vaidya, S. Mahajan, C.M. Preece. - Metall. Trans. 11A (1980), pp. 1139-1147.

[2] D.A. Woodford. - Metall. Trans. 3 (1972), pp. 1137-1145.

[3] A. KaRIMI. - Acta Metall. vol. 37, n 4, (1989), pp. 1079. 1088.

[4] A. Karimi, M. MaAmouri. - Wear, 139 (1990), pp. 149 169.

[5] J.C. HamanN, J.P. Créteur, - P.V. №3682 STLAN MSN (1988).

[6] Stone Marine Manganèse; Technical Brief, Fiche 15, (juin 1977).

[7] J.C. Hamann. - Thèse de Doctorat, ENSM (Ecole Nationale Supérieure de Mécanique) Nantes (déc. 1990).

[8] J.L. HeuzE, A. Karımi. - In « Cavitation », ASME, F.E.D. vol. 116 (1991), pp. 113-118.
[9] C.M. Preece. - Treatise on Materials Science and Techno$\log y$, vol. 16, (1979), p. 298.

[10] A. Karimi. - Mat. Sci. Eng., 86 (1987), pp. 191-203.

[11] J.L. Heuzé. - Thèse Doctorat, Paris VI (oct. 1988).

[12] H. DE LeIrIS. - Métaux et alliages, tome 3, Masson et Cie, (1971), pp. 70-73.

[13] Mateev, Chaikovski et al. - Problemy Prochnosti $n^{\circ} 1$ (1983), pp. 80-84.

[14] K.P. RoEY. - Master's Thesis, Naval postgraduate school, Monterey, California, (sept. 1989).

[15] P.F. Rappeline. - Master's Thesis Naval postgraduate school, Monterey, California (sept. 1989), pp. 339-346.

[16] J.L. Heuzé et B. Dubols. - Journal de Physique, Colloque C3, supplément au $n^{\circ} 9$, tome 49 (sept. 1989).

[17] J.L. Heuzé, F. Gaillard et B. Dubois. - Mémoires et Etudes Scientifiques Revues de Métallurgie (oct. et nov. 1988).

[18] C. Vernot. - Communication interne. 